\title{
O sentido do trabalho na Atenção Primária à Saúde
}

\author{
The sense of work in Primary Health Care \\ El sentido del trabajo en la Atención Primaria a la Salud
}

\begin{abstract}
Leyla Gabriela Verner Amaral Brandão ${ }^{1 *}$, Cristiane Chagas Teixeira ${ }^{1}$, Thaisa Cristina Afonso ${ }^{1}$, Robson Tostes Amaral', Ana Lúcia Queiroz Bezerra'.
\end{abstract}

\section{RESUMO}

Objetivo: Analisar o sentido do trabalho para o enfermeiro na Atenção Primária à Saúde. Métodos: Tratase de estudo de revisão narrativa de literatura, realizada nas bases de dados MEDLINE, LILACS, IBECS, BDENF, CUMED e PUBMED, utilizando como descritores "condições de trabalho", "satisfação no emprego", "recursos humanos de enfermagem", "recursos humanos em saúde", "enfermeiros" e "Atenção Primária à Saúde" nos idiomas português, inglês e espanhol e recorte temporal de publicação de 2000 a 2015 . Resultados: Foram selecionados 21 artigos potencialmente relevantes que abordavam pelo menos um dos seguintes temas: "Papel do enfermeiro na Atenção Primária à Saúde", "Fatores determinantes de Carga de Trabalho", "Repercussões no Trabalho" e "Reflexos na Saúde do Profissional de enfermagem". Considerações finais: Evidenciou-se fatores multivariados que influenciam na vida profissional do enfermeiro e que os mesmos repercutem nos aspectos físicos, emocionais e na percepção do indivíduo em relação aos sentidos do trabalho. Os fatores contribuintes mais relevantes foram a necessidade do acompanhamento da saúde mental, na perspectiva de diminuir as fontes geradoras de desordem na saúde, a fim de propiciar a reorganização do processo de trabalho no âmbito da Atenção Primária à Saúde.

Palavras-chave: Atenção Primária à Saúde, Satisfação no Emprego, Enfermeiros.

\begin{abstract}
Objective: To analyze the meaning of the work for the nurse in Primary Health Care. Methods: It is a study of narrative literature review, carried out in the databases MEDLINE, LILACS, IBECS, BDENF, CUMED and PUBMED, using as descriptors "working conditions", "job satisfaction", "nursing human resources", "Human resources in health", "nurses" and "Primary Health Care" in the Portuguese, English and Spanish languages and publication time cut from 2000 to 2015. Results: Twenty-one potentially relevant articles were selected that addressed at least one of the following topics: "Role of the nurse in Primary Health Care", "Determinants of Workload", "Repercussions in Work" and "Reflexes in Health of the Nursing Professional". Final considerations: It was evidenced are multivariate factors that influence the nurses' professional life and that they have repercussions on the physical, emotional and the individual's perception regarding the meanings of the work. The most relevant contributing factors were the need to monitor mental health, with a view to reducing the sources of health disorder, in order to facilitate the reorganization of the work process in Primary Health Care.
\end{abstract}

Key words: Primary Health Care, Job Satisfaction, Nurses.

1Universidade Federal de Goiás (UFG), Goiânia-GO. *E-mail: leylagabrielawa@hotmail.com 


\section{RESUMEN}

Objetivo: Analizar el sentido del trabajo para el enfermero en la Atención Primaria a la Salud. Métodos: Se trata de un estudio de revisión narrativa de literatura, realizada en las bases de datos MEDLINE, LILACS, IBECS, BDENF, CUMED y PUBMED, utilizando como descriptores "condiciones de trabajo", "satisfacción en el empleo", "recursos humanos de enfermería", "recursos humanos para la salud", "enfermeras" y "atención primaria de salud" en portugués, Inglés y español y la publicación de 2000 del marco de tiempo de 2015. Resultados: Se seleccionaron 21 artículos potencialmente relevantes que abordaban por lo menos uno de los siguientes temas: "Papel del enfermero en la Atención Primaria a la Salud", "Factores determinantes de Carga de Trabajo", "Repercusiones en el Trabajo" y "Reflejos en la Salud del Profesional de enfermería". Consideraciones finales: Se evidenció factores multivariados que influencian en la vida profesional del enfermero y que los mismos repercuten en los aspectos físicos, emocionales y en la percepción del individuo en relación a los sentidos del trabajo. Los factores contribuyentes más relevantes fueron la necesidad del seguimiento de la salud mental, en la perspectiva de disminuir las fuentes generadoras de desorden en la salud, a fin de propiciar la reorganización del proceso de trabajo en el ámbito de la Atención Primaria a la Salud.

Palabras clave: Atención Primaria de Salud, Satisfacción en el Trabajo, Enfermera.

\section{INTRODUÇÃO}

Ao longo da evolução da sociedade, a concepção de trabalho passou por transformações, podendo ser considerado um fator de inserção social, um dos temas essenciais da contemporaneidade, uma vez que tende a ocupar um lugar central na vida dos indivíduos (PERELMAN MD, 2014).

O trabalho pressupõe "uma relação de dupla transformação entre o homem e a natureza, geradora de significado". A partir de uma análise histórica, o trabalho era reconhecido apenas como provedor da sobrevivência, no entanto, ele agrega mais um significado, sendo reconhecido como fonte de realização pessoal (CODO W, 1997). Trata-se de um meio de relacionamento interpessoal, no qual o indivíduo sente parte da sociedade por ter uma ocupação. Desta forma, o trabalho vai além de ser somente um meio de sustento (ARAÚJO MT, et al., 2013). O trabalho possui particularidades, uma vez que seu produto é consumido no ato e em seu desenvolvimento, dependendo do trabalhador e da participação ativa ou passiva do consumidor do trabalho (ARAÚJO MT, et al., 2013), deve ser pautado em questões concretas, mas, principalmente, em atividades onde a prestação do cuidado ao outro seja o centro (BRITO MJM, et al., 2010).

O trabalho em saúde além de ser constituído de um saber científico, tem o compromisso com as necessidades sociais, os atos de produção e o consumo que ocorrem ao mesmo tempo. Na metodologia de trabalho sempre haverá uma interseção entre usuário e profissional, havendo momentos de falas, escuta, responsabilização e criação de vínculos. Nota-se que o enfermeiro está intensamente envolvido nesse processo para o funcionamento do sistema de saúde, pela construção de práticas sociais e de saúde, às quais vêm adquirindo uma repercussão cada vez maior, tanto na educação e promoção da saúde, quanto no fomento de políticas voltadas para o bem-estar social (FEUERWERKER LCM, 2014; BACKES DS, et al., 2012). 
Neste interim, o enfermeiro exerce um papel ímpar na Atenção Primária à Saúde (APS). A APS refere-se a um local cujas relações entre enfermeiro e pacientes são conexas. Ao enfermeiro são delegadas responsabilidades gerenciais das Equipes de Saúde da Família e em alguns municípios até mesmo da Unidade de Saúde da Família de forma geral, o que dificulta ainda mais a organização da agenda de trabalho (MELO RC e MACHADO ME, 2013).

A Atenção Primária à Saúde (APS) é um marco referencial para a organização dos serviços de saúde e tem como proposta a reorientação do modelo assistencial. "A construção do Sistema Único de Saúde avançou de forma substantiva nos últimos anos, e a cada dia se fortalecem as evidências da importância da Atenção Primária à Saúde nesse processo". A APS "é uma forma de organização dos serviços de saúde, uma estratégia para integrar todos os aspectos desses serviços, tendo como perspectiva as necessidades de saúde da população" (BRASIL, 2007).

A Enfermagem é percebida neste contexto como categoria comprometida com o cuidado do ser humano que, adquiri uma consciência que transcende a doença e permite um olhar ampliado sobre o processo saúde-doença-cuidado (SOUSA LB, et al., 2010). A visão ampla e integral proporcionada pelo enfermeiro aos sujeitos, caracterizada como holística, configura-se em um movimento no qual o profissional transpõe para o lugar do outro. $O$ trabalho do enfermeiro pode ser representado pela motivação e prazer em cuidar do outro. Entretanto, pode trazer consigo desgaste pelo acúmulo de funções, que leva ao desprazer, sem que isso implique em incoerência (SOUSA LB, et al., 2010).

Estudo desenvolvido na APS reforça esse achado, apontando que com o acúmulo de funções, o enfermeiro tende a realizar as atividades que requerem respostas mais urgentes, ficando distante da realidade da população. Este fato dificulta a prestação da assistência com melhor qualidade e segurança que, atenda, principalmente, à integralidade das ações, inviabilizando o cuidado longitudinal (BARATIERE T e MARCO N, 2012).

Assim, o estudo tem como objetivo analisar os sentidos do trabalho para o profissional enfermeiro na Atenção Primária à Saúde.

\section{MÉTODOS}

Trata-se de estudo de revisão narrativa de literatura. A coleta foi realizada por meio da bases de dados Medical Literature Analysis and Retrieval System Online (MEDLINE), Literatura Latino-Americana e do Caribe em Ciências da Saúde (LILACS), Índice Bibliográfico Español em Ciencias de la Salud (IBECS), Bases de Dados em Enfermagem (BDENF), Cuba Medicina (CUMED) e National Library of Medicine National Institutes of Health (PUBMED), com corte temporal de 2000 a 2015. Foram utilizados como descritores: "condições de trabalho", "satisfação no emprego", "recursos humanos de enfermagem", "recursos humanos em saúde", "enfermeiros" e "Atenção Primária à Saúde". Esses descritores foram previamente selecionados e realizaram-se diversas combinações e cruzamentos com os mesmos.

REAS/EJCH | Vol. 11 (8) | e528 | DOI: https://doi.org/10.25248/reas.e528.2019 Página 3 de 8 
A primeira seleção dos artigos foi feita pela leitura do título e análise dos resumos, considerando os critérios de inclusão: artigos indexados e disponíveis nas referidas bases de dados, nos idiomas português, inglês e espanhol. Foram usados como critério de exclusão: artigos não disponíveis na íntegra, artigos que não condiziam como o objetivo proposto e artigos que coexistiam na divisão feita em diferentes descritores.

Por fim, foram considerados 21 artigos que condiziam com o objetivo do estudo. Para a discussão dos dados foi articulado com produtos de dissertações e teses de Enfermagem publicadas dentro dos recortes temporais supracitados.

\section{RESULTADOS}

Foram encontrados 580 artigos, sendo que, na base MEDLINE 268 artigos, LILACS 40 artigos, IBECS 25 artigos, BEDENF 13 artigos, CUMED 12 artigos e 222 na PUBMED. E, após a leitura dos títulos e resumos foram selecionados e recuperados 147 artigos. Ressalta-se que dos 147 artigos recuperados, 31 coexistem na divisão feita em diferentes descritores, sendo eliminados 33 artigos que não condiziam com o objetivo proposto, 15 artigos que não estavam disponíveis na íntegra. A organização dos artigos encontrados está representada na Figura 1.

Figura 1 - Fluxograma dos artigos selecionados.

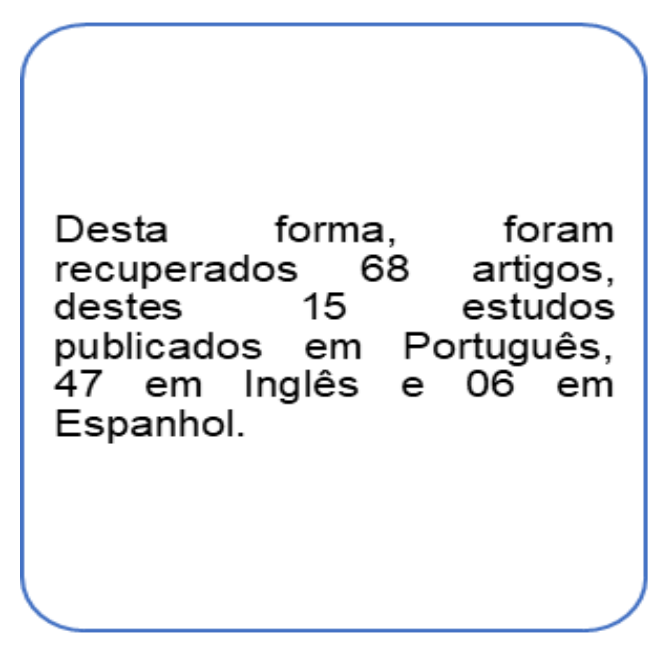

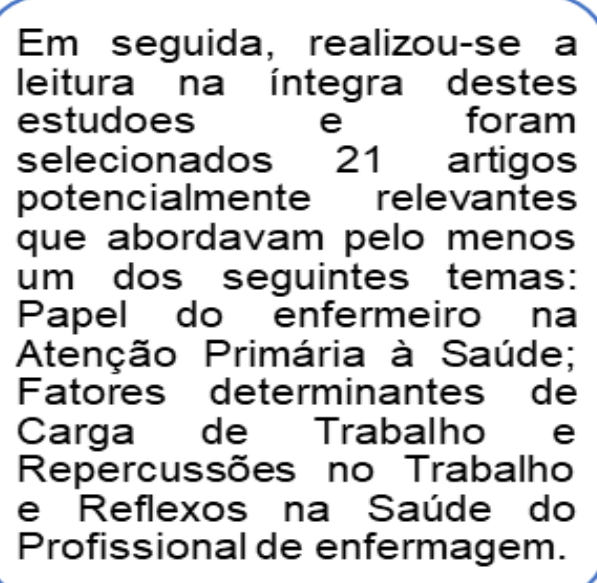

Em seguida, realizou-se a leitura na íntegra destes $\begin{array}{llr}\text { estudoes } & \text { e } & \text { foram } \\ \text { selecionados } & 21 & \text { artigos }\end{array}$ potencialmente relevantes que abordavam pelo menos um dos seguintes temas: Papel do enfermeiro na Atenção Primária à Saúde; Fatores determinantes de Carga de Trabalho e Repercussões no Trabalho Profissional de enfermagem.

Fonte: Dados da pesquisa, 2015.

\section{Papel do enfermeiro na Atenção Primária à Saúde (APS)}

Em dez (10) artigos mencionaram, especificamente, o papel do enfermeiro dentro da APS, tendo como principal atribuição a consulta de enfermagem, assim como, o atendimento de cuidados individuais e coletivos, ações gerenciais e também o profissional inerente ao serviço de saúde. 


\section{Fatores determinantes de carga de trabalho na APS}

Nesta categoria, foram apontados fatores tais como, condições inadequadas de trabalho (10 artigos), desvalorização e falta de reconhecimento profissional relacionado à baixa remuneração (10 artigos), pouco apoio do colega/equipe de trabalho (09 artigos) e situações que representam constrangimento de ordem física e emocional (07 artigos).

\section{Repercussões no trabalho e reflexos na saúde do profissional de enfermagem/enfermeiro}

Em quinze (15) artigos, evidenciou-se que os profissionais apresentaram problemas de ordem psíquica, física, alto grau de exaustão emocional e a presença de stress e Burnout.

\section{DISCUSSÃO}

Ao longo da evolução da sociedade o trabalho conduz a um pensamento de que o empregador desenvolve uma atividade, e como forma de pagamento ele recebe o salário, porém o significado de trabalho não se restringe a este binômio. Ele é um modo de relação social, realização pessoal e de construção da identidade (MAISSIAT GS, 2013).

A estrutura organizacional é importante para que o profissional encontre os sentidos no trabalho desenvolvido, sendo esta uma compreensão necessária para a identificação de sua identidade no contexto do serviço. O setor saúde diferencia-se dos outros setores por ter como uma das características a relação interpessoal (CARAM CS, 2013).

O trabalho em saúde é realizado de forma coletiva, multiprofissional e tem como objetivo à atenção ao ser humano, e para que isto ocorra, existe compartilhamento de saberes, relações entre profissionais e sujeitos na busca de um propósito comum. Nessa lógica, percebe-se que apesar do trabalho em saúde possuir peculiaridades, ele configura com um objetivo comum aos processos de trabalhos (FEUERWERKER LCM, 2014).

Feuerwerker LCM (2014) aponta que o trabalho em saúde além de ser constituído por um saber científico, tem um compromisso com as necessidades sociais (produção de procedimentos, ações educativas e responsabilização) que, resultam no cuidado individual e/ou coletivo. O "mundo" do trabalho é complexo, composto de intensas relações e envolve campos da micropolítica do trabalho vivo em ato, sendo este representado pelo modelo usuário centrado (MERHY EE, 1999).

A Atenção Primária à Saúde (APS) é um marco referencial para a organização dos serviços de saúde e tem como proposta a reorientação do modelo assistencial. "A construção do Sistema Único de Saúde (SUS) avançou de forma substantiva nos últimos anos, e a cada dia fortalece as evidências da importância da Atenção Primária à Saúde nesse processo". A Conferência de Alma Ata, em 1978, enfatizou a Atenção Primária à Saúde como estratégia fundamental para a substituição do modelo tradicional de atenção à saúde, com destaque para a Carta de Ottawa, de 1986, e que vem se enriquecendo com as declarações internacionais posteriores (BRASIL, 2007)

REAS/EJCH | Vol. 11 (8) | e528 | DOI: https://doi.org/10.25248/reas.e528.2019 Página 5 de 8 
Os profissionais inseridos na APS são os que estão na linha de frente no atendimento aos usuários que procuram o SUS, e para atender a complexidade de exigências populacionais e das diretrizes do serviço, o trabalhador está susceptível a diversas formas de prazer e alguns fatores negativos determinantes a carga de trabalho (MAISSIAT GS, 2013).

A Enfermagem é percebida como uma categoria comprometida com o cuidado do ser humano tecendo maior vínculo junto à população. O Enfermeiro é fundamental para o desenvolvimento das ações de saúde, sejam elas de forma direta, como a consulta de enfermagem, práticas assistenciais e gerenciais; ou indireta, no momento de escuta do usuário, na realização da educação em saúde para o usuário, embora sejam muitas vezes subutilizados (TODD C, et al., 2007).

O trabalho deste profissional vem permeado de desafios levando em consideração, ao mesmo tempo, as necessidades da organização de saúde, assim como, dos usuários dos serviços de saúde. Nessa lógica, fica claro que o enfermeiro possui uma gama de atribuições, abarcadas por tomadas de decisões e planejamento. Nota-se ainda que, em algumas ocasiões, devido a estas multifaces de atuação, pode ocorrer um acúmulo de atribuições e responsabilidades que podem comprometer a qualidade do serviço prestado e refletir no processo de sofrimento para este profissional, resultando em uma desordem de cunho psíquico ou física (TODD C, et al., 2007).

A realidade do trabalho dos enfermeiros em APS, a exemplificar a Estratégia Saúde da Família (ESF), é diversa das atribuições previstas e, por vezes, acaba assumindo responsabilidades e funções além dos recursos de que disponibilizam para sua prática, acumulando atribuições que interferem na qualidade do serviço e no desenvolvimento das atividades. Sim J (2015) adiciona a necessidade de estratégias desenvolvidas pelo enfermeiro para assistência segura e de qualidade no âmbito do cuidado.

Estudo aponta os aspectos negativos presente no cotidiano do trabalho do enfermeiro que interferem na saúde física e/ou emocional deste profissional, de acordo com Gil CRR (2006) baixa remuneração, sobrecarga de trabalho, pouca valorização do enfermeiro pela chefia e instituições, entre outros são apontados como aspectos negativos dentro do processo de trabalho.

O enfermeiro ao buscar ter mais reconhecimento, responsabiliza por diversas funções, atividades em excesso, distanciando da assistência direta, o que gera sentimentos negativos, como a fragilidade em sua identidade profissional (BAGGIO MA, 2006 ; VIEIRA I, 2013).

Percebe-se que a organização do trabalho pode ter influência no estado de saúde do profissional, como insuficiência de recursos para desenvolver o trabalho, o que ocasionam danos físicos e cognitivos (SHIMIZU HE e CARVALHO JUNIOR, 2012). Apesar dos resultados evidenciarem fatores negativos que influenciam na carga de trabalho, nota-se aspectos positivos, conforme aponta Gil CRR (2006) formação de vínculo com o paciente, processo de trabalho em equipe, conhecer as condições de vida e de saúde da população, autonomia profissional, entre outros. O trabalho do enfermeiro pode ser representado pela motivação e prazer em cuidar do outro, mas pode trazer consigo desgaste pelo acúmulo de funções, que leva ao desprazer, sem que isso implique em incoerência (BAGGIO MA, 2006 ; VIEIRA I, 2013).

REAS/EJCH | Vol. 11 (8) | e528 | DOI: https://doi.org/10.25248/reas.e528.2019 Página 6 de 8 
No que tange, as repercussões na saúde do trabalhador, muitos enfermeiros desenvolvem alterações da saúde mental, experimentando situações de sentimentos de exaustão, stress, desmotivação, Burnout ou esgotamento profissional, além de manifestação de problemas de ordem físicas. No campo da saúde do trabalhador, a construção do conhecimento de possíveis predisposições desencadeantes da síndrome do esgotamento profissional, em especial nos enfermeiros, atua em diferentes ambientes de trabalho, como em condições inadequadas, e em situações de desgastes físico, biológico e psicossocial (ROSSI SS; et al.,2010).

Estudo brasileiro evidenciou a prevalência da Síndrome de Burnout de 6,7\% a 10,8\%, sendo os fatores associados a carga horária de trabalho excessiva e a insatisfação profissional (SILVA SCPS, et al., 2015). Tais fatores são causas de absenteísmo e pedido de licença, que gera impactos na qualidade do serviço prestado e desencadeamento de outros problemas deste cunho (ROSSI SS, et al.,2010)

Ao analisar os resultados, podemos basear nas seguintes dimensões: "Dimensão Organizacional", "Dimensão Individual" e "Dimensão Social" (CARAM CS, 2013). Na dimensão organizacional foram abordados aspectos como: condições inadequadas de trabalho e relacionamento interpessoal. Na dimensão social, emergiram fatores como: insatisfação com o trabalho, falta de reconhecimento do profissional enfermeiro, baixa remuneração; e por fim, na dimensão social, relaciona-se o baixo valor social do trabalho e a inserção do trabalho na saciedade. Visto que, ambas as dimensões aparecem como determinantes para a insatisfação do trabalho, podendo ocasionar em alguns resultados não satisfatório para o profissional, organização e paciente.

\section{CONSIDERAÇÕES FINAIS}

No estudo emergiram fatores multivariados que influenciam na vida profissional do trabalhador, e que os mesmos repercutem nos aspectos físicos, emocionais e na percepção do indivíduo em relação ao sentido atribuído ao trabalho. Um trabalho que possui sentido para enfermeiros que atuam na Atenção Primária à Saúde, precisa possuir algumas características, dentre as quais destacam-se: ser fonte de prazer e de relações interpessoais (profissionais e sociais), salário adequado às atividades, propiciar qualidade de vida no trabalho, reconhecimento profissional e carga de trabalho adequada. Destaca-se que a estrutura organizacional é de fundamental importância para que o profissional consiga encontrar sentidos no trabalho.

\section{REFERÊNCIAS}

1. ARAÚJO MT, MONTENEGRO LC, ALVES M, et al. O significado do trabalho para os profissionais de um serviço substitutivo de saúde mental. Rev Esc Enferm USP. 2013;47(3):664-70.

2. BACKES DS, BACKES MS, ERDMAN AL, et al. O papel profissional do enfermeiro no Sistema Único de Saúde: da saúde comunitária à estratégia de saúde da família. Ciência \& Saúde Coletiva. 2012;17(1):223-30.

3. BAGGIO MA. O significado de cuidado para profissionais da equipe de enfermagem. Revista Eletrônica de Enfermagem. 2006;8(1):9-16. 
4. BARATIERI T, MARCO N SS. Longitudinalidade no trabalho do enfermeiro: identificando dificuldades e perspectivas de transformação. Texto Contexto Enferm. 2012;21(3):549-57.

5. BRASIL. Ministério da Saúde. Conselho Nacional de Secretários de Saúde. Atenção Primária e Promoção da Saúde. Brasília: CONASS; 2007.

6. BRITO MJM, VIANNA PCM, SILVA TC, et al. O significado do trabalho para profissionais de saúde mental. J Health Sci Inst. 2010;28(2):165-68.

7. CARAM CS. Os sentidos do trabalho para profissionais da saúde do CTI de um Hospital Universitário. Dissertação. Universidade Federal de Minas Gerais/ UFMG, Belo Horizonte; 2013.

8. CODO W. Um diagnóstico do trabalho (em busca do prazer). In: TAMAYO A, BORGES-ANDRADE JE, CODO W (Org.). Trabalho, organizações e cultura. São Paulo: Cooperativa de Autores Associados; 1997. p.21-40.

9. FEUERWERKER LCM. Micropolítica e saúde: produção do cuidado, gestão e formação. Coleção Micropolítica do Trabalho e o Cuidado em Saúde. Porto Alegre, 1a ed., editora Rede Unida; 2014.

10. GIL CRR. Práticas Profissionais em Saúde da Família: Expressões de um Cotidiano em Construção. Tese. Escola Nacional de Saúde Pública Sérgio Arouca, Fundação Oswaldo Cruz. Rio de Janeiro; 2006.

11. MAISSIAT GS. Prazer e sofrimento de Trabalhadores da atenção Básica à saúde da Psicodinâmica do Trabalho. Dissertação. Universidade Federal do Rio Grande do Sul/UFRS; 2013.

12. MELO RC, MACHADO ME. Coordenação de unidades de saúde da família por enfermeiros: desafios e potencialidades. Rev Gaúcha Enferm. 2013;34(4):61-67.

13. MERHY EE. O ato de governar as tensões constitutivas do agir em saúde como desafio permanente de algumas estratégias gerenciais. Ciênc saúde coletiva. 1999;4(2):305-14.

14. PERELMAN MD. Viviendo el trabajo. Transformaciones sociales, cirujeo y venta ambulante. Trabajo y Sociedad; 2014.

15. ROSSI SS, SANTOS PG, PASSOS JP. A Síndrome de Burnout no Enfermeiro: Um Estudo Comparativo entre Atenção Básica e Setores Fechados Hospitalares. R pesq.: cuid fundam online. 2010;2(4):1232-39.

16. SILVA SCPS, NUNES MAP, SANTANA VR, et al. Burnout syndrome in professionals of the primary healthcare network in Aracaju, Brazil. Ciência \& Saúde Coletiva. 2015;20(10):3011-20.

17. SIM, J. Measuring the quality and safety outcomes of nursing practice, Doctor of Philosophy thesis, School of Nursing, University of Wollongong; 2015.

18. SHIMIZU HE, CARVALHO JUNIOR DA. O processo de trabalho na Estratégia Saúde da Família e suas repercussões no processo saúde-doença. Ciênc saúde coletiva. 2012;17(9):2405-14.

19. SOUSA LB, TORRES CA, PINHEIRO PNC, et al. Práticas de educação em saúde no Brasil: a atuação da enfermagem. Rev Enferm UERJ. 2010;18(1):55-60.

20. TODD C, HOWLETT M, MACKAY M, et al. Family Practice/Nurses in Nova Scotia. Can Nurse. 2007;103(6):23-7.

21. VIEIRA I. Uma análise original da fadiga no trabalho. Physis. 2013;23(4):1359-68. 